\title{
Leveraging BIM and Blockchain for Digital Twins
}

\author{
$1^{\text {st }}$ Yasin Celik \\ BRE Institute of Sustainable \\ Engineering, School of Engineering, \\ Cardiff University \\ Cardiff, UK \\ celiky@cardiff.ac.uk
}

\author{
$2^{\text {nd }}$ Ioan PETRI \\ BRE Institute of Sustainable \\ Engineering, School of Engineering, \\ Cardiff University \\ Cardiff, UK \\ petrii@cardiff.ac.uk
}

\author{
$3^{\text {rd }}$ Yacine Rezgui \\ BRE Institute of Sustainable \\ Engineering, School of Engineering, \\ Cardiff University \\ Cardiff, UK \\ rezguiy@cardiff.ac.uk
}

\begin{abstract}
The digitalization of the construction industry has been supported recently with various building information modelling (BIM) methods applied to lifecycle stages. This has enabled the transition towards an improved management of information required in the development of different construction project tasks. In a digital construction context, traceability of models and collaboration across disciplines is required especially when dealing with sensitive data and critical infrastructures. Blockchain technology has the potential to advance the automation of processes in the construction industry by offering a higher level of data traceability, incentivising participation, and ensuring transparency with projects. This paper presents how BIM and Blockchain can be utilised to facilitate the establishment and implementation of Digital Twins for construction projects. A highway construction case study is used for demonstrating the implementation of smart contracts for managing tasks and processes across different disciplines involved in a construction project.
\end{abstract}

Keywords-Digital Twin, BIM, Blockchain, Construction, Collaboration, Smart Contracts;

\section{INTRODUCTION}

Industries and sectors have started to increasingly adopt digital solutions for managing their core processes and save costs based on the recent development in information technologies [1]. Such technologies have brought great opportunities for the creation of digital inventions in physical assets and, in general, a higher order of intelligence in managing such assets [2].

In the construction industry, the advent of Artificial Intelligence (AI), Internet-of-Things (IoT) and Blockchain technologies have provided new methods and techniques to sustain projects throughout the lifecycle stages. Such solutions can support with the conceptualization, prototyping, testing and design optimization period, but also throughout the operational phases of the life cycle. Although the significance of numerical modelling methods and experiments cannot be questioned in the first analysis phase of a construction project, the opportunity for real-time data availability in the operating phase provides new opportunities for tracking and optimizing processes during a project life cycle [3]. The seamlessly integration of data from such project stages including models based representation with numerical analysis can be achieved via digital twinning.

Digital twins have emerged as digitized virtual representations of real physical assets. The creation of a digital twin necessitates dependable management and monitoring of data including storage and analysis with adequate security policies. The Blockchain can be used to provide several security features to a digital twin. Such services include traceable, tamper-proof, and encrypted services implemented by smart contract interactions. [4].

A digital twin for construction consists of the following key parts: Physical layer, Digital layer, Cyber layer. The physical layer defines objects, assets, materials, personnel, infrastructure, facilities, systems, or resources such as the objects that tend to have material assets in the physical world [5]. The digital layer identifies the development, modification, evaluation, optimization, or analysis of a static, dynamic model and real-time data by storing data in original or different file formats such as Computer Aided Design (CAD) or Industry Foundation Classes (IFC). Prior to creating physical assets, digital models are created in a storage digital environment [5]. Validation and verification functions, as well as sharing and updating information between parties, are all supported by Blockchain. The cyber layer, on the other hand, provides computing and storage capabilities for creating a complex data model that can scale technological infrastructure. In addition, the data model leverages on Blockchain, IoT, Big Data, and Cloud technologies to generate information and expertise for a number of Digital Twin-enabled implementations [6].

The construction industry has begun the digitalisation process with Building Information Modelling (BIM), which brings features such as detailed design, conflict detection and energy analysis to the sector. BIM was designed with the intention of creating a single source of proof for stakeholders, including clients, designers, developers, contractors, and subcontractors. While BIM could have multiple contributors to create or develop design models, the digital twin transition required more advanced data analysis and integration to support a variety of actionable insights. [7].

In recent years, various digital twinning technologies are presented in a range of industrial and research studies such artificial intelligence and robotics, big data and analytics, virtual and augmented reality, advanced BIM implementation, cloud and edge computing with the objective to advance the digitization of the construction industry [8]. Such digitalization has emerged with the need of information sharing, and data security required in different multi-stakeholder systems. Some construction issues, such as the fake concrete experiments on the Hong Kong-ZhuhaiMacau bridge, were also addressed by using Blockchain technology to protect information sharing or to be proof for forensic investigation [9]. 
Building Information Modelling has been demonstrated as an efficacious technology for achieving digitalization in the construction sector across the life cycle of a building [10]. BIM facilitates collaboration for digital twinning strategies requiring the same level of "trust" in the networked system [11]. As data must be stored and exchanged in a secure and safe environment, technology should be well-designed in order to ensure efficiency and security [12]. The combination of BIM with emerging technologies such as Blockchain, Artificial Intelligence, Cloud Computing and Machine Learning provide significant digital transformation opportunities for the building industry. As a computing level, cloud computing and Blockchain also facilitate real-time creation and coordination of a visualized database, enabling more effective and secure interdisciplinary collaboration [13].

In this paper we argue that digital twinning strategies should rely on a seamless integration of Blockchain and BIM systems for a smarter construction industry. Digital twinning strategies have the ability capture, therefore, contextual aspects embedded into BIM, complemented with security and automation advantages that Blockchain can bring for assisting in the management of construction workflows.

This study aims to explore, develop and implement a decentralized Blockchain-based solution with a view to deliver a reliable, trustworthy, sustainable, and efficient system to track and trace the various phases involved in the development of digital twin. This paper contributes to existing digital twinning research agenda by developing a viable approach that includes Blockchain into the BIM lifecycle of the construction process with the objective to go beyond the digital twinning theory.

The rest of the paper is organised as follows: Section 2 provides a few relevant case studies in the digital construction area. The methodology used to perform our research is discussed in Section 3. Section 4 discusses and develops a Blockchain and BIM model for the digital twins with regards to building life cycles. In Section 5, a real-world case study using Blockchain and BIM involving a multidisciplinary construction project is used to validate the paper. The outcomes and future work are discussed in Section 6.

\section{BACKGROUD AND CONCEPTUALISION}

In this section we present several key technologies supporting the development of digital twins with an emphasis on BIM and Blockchain.

\section{A. Digital Twin}

A digital twin represents objects or subjects in the digital world with their data, roles, and communication capabilities [3]. The digital twin is a digital model that is a dynamic representation of an entity that mimics its real-world behaviour based on data [5]. For example, a digital representation of an actual building is used in the BIM process during the entire lifecycle, from construction planning to building activity, to ensure optimized communication and sharing of knowledge between all participants and discipline involved in a construction project[6].

A digital twin, therefore, operates with real-time data capture by sensor systems and analyses real-time structural and environmental parameters of a physical object in order to conduct highly accurate digital simulations and analytics. For the digital twin, BIM provides the integration of cost estimation and time sheet data to improve the efficiency of a construction project. A digital twin is designed to combine the collected data in real time and optimize the building's interaction with the environment and users [14]. The main difference between a virtual simulation and a digital twin is the reliance on physical properties of an entity, sensor networks and real time data based on which a digital twin is developed [15].

\section{B. Building Information Modeling (BIM)}

It has been developed from the need to integrate the building's 3D computer-aided design models with supplementary details on construction features, schedule, expense and maintenance control, minimizing costs while eliminating design and construction errors. It is used in architectural engineering and facility management functions for visualization and consistency of design, conflict detection, cost and time calculation, and to make stakeholder interoperability more effective [16].

BIM is constantly changing, starting with the 1990s Level 0 BIM that was mostly done with paper drawings. In the 2000s, companies started using 3D CAD modelling in BIM Level 1, and a common data environment (CDE) was used for digital data sharing, but without the ability to share models between participating members. Level 2 BIM was characterised with collaboration and sharing of digital files and models and the use of common file formats Industry Foundation Class (IFC). Level 3 BIM is integrated with a focus on collaboration between stakeholders and is stored in a central cloud-based repository to enable collaboration across the lifecycle of the construction. Nowadays, during facility design, construction and knowledge transfer, BIM is often used to increase resource efficiency with the aim to minimize and eliminate errors in building operations[17].

Whereas, BIM is not intended to work with real-time data, although used for designing, building, and maintenance activities and inter-operability in industry, a digital twin is a physical asset's digital equivalent that functions as a dynamic data based BIM network [18].

Although the outcomes of the architecture, engineering and construction (AEC) digitalization are promising, adopting BIM across the sector has actually shown several limitations [19], with additional cybersecurity threats arising in this emerging digital context. The emergence of new digital technology provides opportunities for digital inventions but the network-based data sharing with BIM and the common data environment makes the industry vulnerable to potential cyber-physical attacks [20].

\section{Blockchain}

A BIM workflow is based on a centralised database that often exposes privacy and accessibility risks including data breaches identifying significant risks for compromising data privacy, quality and integrity such as GDPR [21]. To tackle such risks, Blockchain is a reliable solution for construction that offers clear and precise distribution of information between participants by assisting information control. A distributed data recording and maintenance scheme is part of the Blockchain known also as the Distributed Ledger Technology (DLT). In a Blockchain context, participants can verify and approve the autonomy while updating information contained in the blocks. For a digital twin the integration between BIM and Blockchain can address several limitations 
and problems, including model specification, adaptation, and error liability [22].

Blockchain can be delivered also as "smart contracts" which are computer protocols that seek to eliminate the need for contractual intermediaries by initiating, validating, or enforcing contract agreements or success within a project [23]. In a construction project, the ultimate aim of a smart contract is to meet traditional contract conditions (such as payment terms), minimize requirements, and eliminate the need for reliable intermediaries [24]. Smart contracts are structured on a code structure that runs between blocks to improve the protection of the Blockchain application by defining a number of fields including address, functions, status, and also enabling code execution [25]. Blockchain can allow users of BIM to share and exchange data while providing better transparency and operating practices, with a view to advance the delivery of a digital twin for various construction scenarios.

As a limitation, Blockchain is subjective to environmental and sustainability policies due to its intensive energy consumption for application involving mining (i.e. Bitcoin) [26]. Blockchain operates on data via an open web transactions logs under the permissionless architecture which also raises privacy concerns [27].

\section{Cloud}

In the construction industry, cloud computing is a technology that can reduce processing load while supporting the digitalisation [24], [28]. Reducing equipment and costs, public facilities and utilities, reliability with a fully automated method, and simple scalability are just a few of the advantages. Clouds provide flexible IT infrastructures while ensuring connectivity to the Internet for smart applications. Despite the fact that the cloud offers many valuable resources, organizations are slow in adopting due to privacy concerns, security concerns and cloud challenges [29]. Although service providers work as third parties with strict policies, privacy rules may be with reduced efficiency comparing to those enforced by the organizations themselves. As cloud processing involves outsourcing of data on a third party infrastructure, risks appear around data access and control. Furthermore, privacy concerns around personal information also represent key aspects for data cloud storage [30]. Therefore, several potential risks with cloud technology arise in construction projects especially when private and commercial information such as project cost are shared across disciplines [31]. Data exchanges amongst construction team members is also another point to consider where the users connected to the network have access to client or organization projects information. In addition, the energy consumption affected with computation for storing and maintaining unnecessary data is another limitation on this process [32].

The combination of Blockchain technology and cloud computing addresses digital safety and security and service availability. Blockchain eliminates the majority of cloud limitations by using the cloud sites as nodes for deployment of various protocols and transactions. Since all nodes on the same platform can share data, each node has a record of the interactions while improving flexibility. The Blockchain network converts all block records into a hash code and provides a cryptographic signature for each block utilizing encryption algorithm. Thus, without any interruption, the data stored in the cloud is replicated on Blockchain nodes, optimizing data efficiency and data validity. In a construction project, the discipline privacy is also guaranteed by Blockchain, and the record can be securely encrypted from the system to prevent third-party access to the data [33]. In addition, the cloud and the Blockchain can be used with BIM to help the building sector in becoming sustainable and more functional while making a valuable contribution to the digital transformation of the construction industry. Although widely explored in several studies focusing on the combination of these technologies, this paper demonstrates the benefits and application areas in BIM and Blockchain from a digital twin perspective based on a real construction case study.

\section{RELATED WORK}

In the field of Building Information Modeling (BIM) and Blockchain, there are several research projects that seek to establish a methodology for the use of Blockchain in the construction industry with the view to prevent delays, minimize costs and create a secure environment for construction stakeholders. In general, the lack of a specified framework for the digital twin has made technical advances including human and environmental factors more important to the construction industry's needs.

The digital twin technologies are developed systematically with a view to offer smart solutions in the construction sector. As a digital twin is a digital representation of a system or a physical asset, it allows efficient data analytics and effective decisions with the facility to combine data from multiple data sources. Digital twins can advance the Building Information Modeling (BIM) technologies supporting fault identification and diagnostic problems in the built environment. Digital twin technologies have been developed also to maximize efficiency, reduce failure rates, shorten production schedules, and open new market opportunities. There are not many twin applications aimed at improving the safety of process plant operators and maintenance staff [34].

High levels of stakeholder participation and the implementation of building technologies require an integrated approach in order to achieve collaboration among individuals and technology. BIM offers a convergent solution that makes project data available to multiple construction technology ecosystem use cases, such as design and data management, simulations, and project scheduling [35]. One BIM challenge is determining responsibilities and rights due to similarity in roles and responsibilities. Other issues include intellectual property security, risk allocation, safety and security and trust in third parties, and the availability of technology intermediaries [36].

With the combination of BIM and Blockchain, the information forming the source of physical, digital and application resources can be verified transparently and stored publicly or privately. With Blockchain, a collectively chosen validator updates this distributed ledger one block at a time. For a construction project, the Blockchain consensus mechanism selects a leader discipline and ensures that the chosen leader has sufficient credentials, and that each node accepts the leader's block as a suitable block to be included in the Blockchain [37]. The Blockchain database prevents any alteration in data and copyrights, since the same information entered and validated in the chain is distributed and then stored across all nodes. Blockchain performs this verification and process independently from third parties [38]. The development of decentralized Blockchain 
technology has been seen as a reliable alternative to information storage, data processing, functioning and trust, making it ideal for high security requirements for critical digital infrastructures. While different industries are currently researching and studying Blockchain technology in their applications, the research in the construction sector is still developing [39].

Blockchain has the ability to streamline and accelerate design works and engineering practices, with numerous advantages for the business and disciplines. Such advantages include increased flexibility to team members to collaborate while ensuring performance, cybersecurity, and data interchange integrity. Blockchain could lead to more efficient governance and the use of a variety of technologies to increase efficiencies, change organizational environment, and enable adoption of digitalisation. For instance, a cloudbased technology can enable the development and coordination of a visualized database in real time, as well as serving as a platform for multi-disciplinary coordination. Smart contracts, on the other hand, are also a series of preprogrammed procedures that can be executed automatically when certain conditions are fulfilled. Distributed wireless sensor networks have become beneficial in construction projects, enabling the adoption of Blockchain to improve IoT frameworks by reducing constraints and maximizing its efficiency [40]. As it is difficult to maintain trust among stakeholders in a developing building industry, the resulting time loss and process costs and relationships are needed as to address information sharing in highly complex workflows [41]. Smart contracts are self-executing programmes that define the rules and responsibilities that belong to a contract and allow the automatic implementation of specifications in project tasks. They can be enforced in a Blockchain ledger and can save time and resources by reducing administrative functions and avoiding transactional conflicts [42].

BIM supports various project specifications, providing the basis framework for the realisation of a construction digital twin. Digital technologies are widely adopted in the construction section ranging from Internet of Things, Artificial Intelligence, and other technologies. Such technologies ensure a high level of connectivity while facilitating smarter construction services, better automation, and improved knowledge sharing and cyber security issues impacting large-scale infrastructures [18]. The integration of BIM with Blockchain represented an opportunity to assist the construction stakeholder to solve the issue of transparency and increase the performance of the overall building project by offering benefits such as value transfer for digital assets, digital identification, certificate facilities, and security [43]

In previous studies, Nader \& Jameela [44] propose a middleware Blockchain approach to support smart manufacturing applications with Blockchain services to enable more secure, reliable, traceable and autonomous smart manufacturing applications. The solution is developed as a cloud-integrated architecture by combining it with Blockchain technology [33]. Hasan et al. [4] propose a Blockchain-based digital twin system that ensures reliable and transparent transaction, and data provenance track-andtrace, availability, and immutability. Smart contracts are used to rule and monitor transactions initiated by participants in the development of the digital twin.

Kim and Laskowski [45] have introduced a Blockchain method including data source tracking, traceability, transparency, and tamper-proof logs to meet the requirements of supply chain industries. These applications prove that Blockchain is a highly secure distributed ledger that can be trusted in a construction project. Blockchain serves as a central platform for all stakeholders' activities and interactions, ensuring transparency and accountability [46].

\section{DIGITAL TWIN METHODS FOR CONSTRUCTION}

We have conducted a thorough literature review using academic and conference papers, textbooks, technical guides, and industrial resources to better understand the use of Blockchain technology in digital construction. As reported by studies, automation and better connectivity solutions for the construction industry can be provided by combining IoT, Blockchain, and machine learning. Low protection, reliability, maintenance, usability, and costs are major issues. Blockchain technology tackles issues such as transparency, energy efficiency, power, reliability, and maintenance [47].

Digital twins are increasingly discussed and adopted based on their ability to support modelling, forecasting and optimisation on various digital artefacts [5]. Privacy and security, and also the reliability of data sources are some of the major challenges when using digital documents and data [48]. As Blockchain implementation throughout the building life cycle can accelerate the construction industry's current digitization process, it is important to define benefits that a digital twin can provide for various project stages.

Figure 1 shows the contribution provided to digital twin via Blockchain integration over BIM Riba stages. As reported by our literature review and informed from the case-study presented in the evaluation, digital twin contributions to the construction sector based on BIM and Blockchain are as follows:

Real-time remote monitoring and management: A digital twin can provide an in-depth perspective of digital assets which can be controlled remotely using feedback systems. All activities can be kept in a record of data changes with a distributed database allowing decisions to be made adequately with real-time actionable insights [49].

Better efficiency and security: A digital twin can bring better security and governance where machines can be actuated remotely and users can undertake inventive work in a healthy and stable environment [11].

Maintenance and planning estimation: Multiple sensors monitoring physical assets may produce large quantities of data in real time for an extensive digital matching. With the smart analysis of the results and performance monitoring, flaws in the device can be identified much earlier. Such solutions save time by delivering faster performance for maintenance and licensing [50].

Case and risk evaluation: What-if analysis are possible with a digital twin leading to improved risk management. Such analysis can evaluate the system reaction as well as subsequent coping mechanisms in order to address unexpected circumstances [51].

Collaboration: With BIM and Blockchain, teams can improve synergies and alliances via collaboration leading to enhanced productivity with greater autonomy and preventing delays while eliminating third parties [52].

Immutable Data Transactions: Blockchain allows the tracking of model changes and provenance model records 
with additional timestamps for validation and re-assembling for different digital artefacts within a construction project [53].
More effective and predictive analytics: The availability of actual predictive data and visualization tools can enable digital twins to support decision-making for various construction specific applications [54].
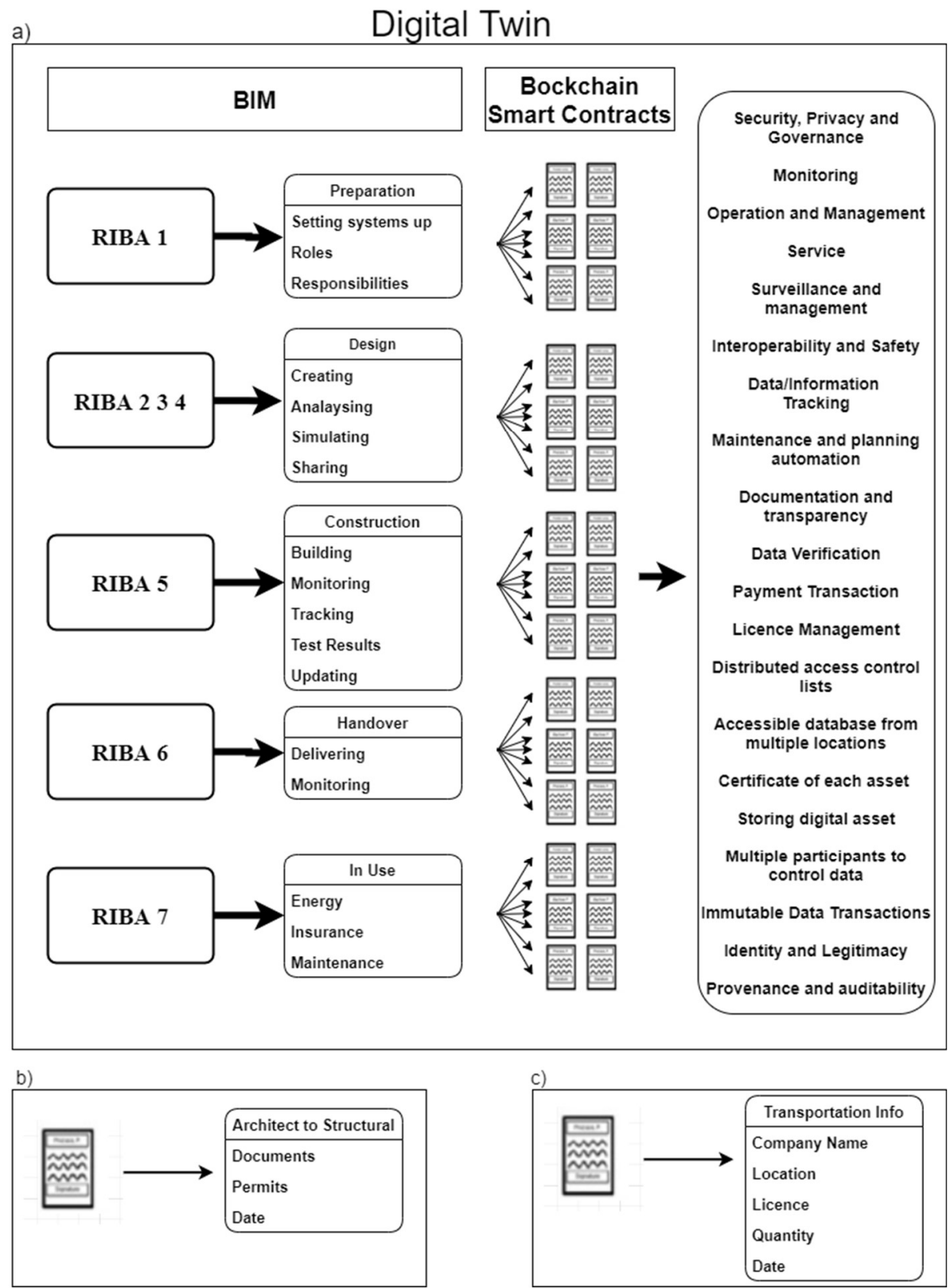

Fig. 1. a) BIM and Blockchain integration for digital twin b) Example of the data record in data exchange c) Example of the data record with its features on Blockchain

Material and services customization: The demand for customized products and services is anticipated to increase with comprehensive historical data, expectations of different stakeholders and evolving business dynamics of competitors. A digital twin would allow quicker and simpler solution to accommodate evolving demands in the perspective of future software defined factories [55].

Better documentation and transparency: Disciplines and users can see the transactions on a Blockchain network, but they cannot change data already stored [56]. Combined with automatic monitoring, real-time details can help disciplines to have updated digital replica in order to increase transparency [57].
Data/Information tracking: Blockchain provides a framework for tracing file signatures across platforms, ensuring traceability in the design and review process within a digital framework [58].

Immutability: Security breaches are limited since the Blockchain is an immutable and open network. Node replication is used where each node in the system has a copy of the digital ledger [59]. When the intermediary blocks are being transferred between software tools, the Blockchain channel ensures that files are not tampered.

License management: The Blockchain can ensure a digital component integrity by functioning as a unifying 
network where transactions can be registered and tracked on a fragment-by-fragment basis using a serial number / digital identity. Append-only functionality is also available on the Blockchain as a mean to check the steps involved in certification and qualification [58].

Decentralization: Disciplines and users can access the Blockchain network and store shared data using private keys based on a decentralized mechanism [60]. The network is managed by a community of nodes, thereby eliminating the restriction associated with a central authority.

Multiple participants to control data: Construction disciplines have access to the Blockchain network to validate a process history and associated data, as well as ability to track various iterations of a process [53].

Automation: Contracts are converted into smart contract with self-executing codes and the entire construction process is controlled automatically. This avoids any potential delays by ensuring that the procedure is completed timely [61].

\section{EVALUATION}

The evaluation was performed with data and models from the Clouds4Coordination (C4C) project [62] involving multidisciplinary collaboration for a highway construction project. The framework was developed using a cloud computing infrastructure [63] to support a real-world UK highway development project involving the construction of a new bridge. We present below a Blockchain-based framework integrating BIM models within the design phase of the construction life cycle.

\section{a. Scenario description and assumptions}

The project involved the collaboration between multiple disciplines which are exchanging BIM data (IFC objects) during various project tasks. In the design phase which is initiated by the Architect, structural objects such as columns in the building are prepared and designed and shared with a structural engineer. Structural engineers analyse, calculate, and dimension the shared design which is reviewed by a contractor and then detailed and built by a specialist manufacturer.

The project identifies in the first phase the Architect that can place an object in the design as a generic icon to represent a structural column in the initial design phase. After calculating the requirements for the column, a real-size column can be placed in a BIM model by the structural engineer. The architect then positions real-size column boxes to describe the visibility of the generic columns in order to illustrate the structural engineers' model taking into account the relations with other structural elements on the project. This process is repeated by other design teams until the design process is completed. Each party needs to take responsibility for the design process, which may have an impact on the project's overall performance.

The Architect cannot move the column to meet the spatial requirements because this can affect the size and material requirements for both the columns and the elements it supports. Similarly, the structural engineer and other design team cannot move the column as this may affect the functional requirements of the space around the column. All these project tasks necessitate synchronisation of the procedure to be executed in a coordinated manner. Interdisciplinary teamwork, trust, and collaboration are the important aspects impacting the design process. In construction projects, the cause for revisions or conflicts, as well as any problems that occur, often results in additional delays and costs.

The cloud-based federated model from the $\mathrm{C} 4 \mathrm{C}$ project encourages each party to build their own records within their own continuing workspace to exchange with the design team until the data has been integrated. This makes for a consistent audit trail and everyone is aware of their particular design professions based on a protocol for implementing changes. While each dataset has its own ID, every issue can be detected and connected to the relevant party. Any modifications can be tracked and trusted by architects, structural engineers, and other design teams. As a result, the process's development, roles and responsibilities, and interdisciplinary collaboration will all be feasible in a digital environment.

When the data is coordinated in the design stage, the Blockchain-based federated model solution within the BIM environment generates its own documentation, which is then shared with the design team. This allows for a transparent automated process since everyone understands their basic design responsibilities and the protocol for improvement, as well as the importance of recording all activities on the Blockchain database as trustworthy data records.

Since each dataset has its own identity, any problems can be identified and linked to the correct discipline. At the file, layer, and object level, each BIM model classifies elements with metadata identifying the issuing discipline. This creates a complex filtering system inside models for detecting coordination issues across various disciplines and making them transparent to the public to increase efficiency. When a discipline or user logs into the system, all activities which has been done, are registered into the Blockchain as a proof of record marked with a timestamp.

As mentioned above, a change in the position of a column will require updating the Architects, Structural Engineers, and Fabricators models, as well as resolving the consequences with the rest of the design and construction team. The platform checks are used to ensure that data is synchronized when it is released and recorded to the Blockchain as an immutable data record. Thus, if a comprehensive assessment of publicly available data is performed, the necessary data and information is securely retrieved. The structural engineer coordinates the dimensioned structural column and divides it as needed to demonstrate the design evolution and recognizing the responsible party in the event of coordination issues. As a result, all contributions made during the process are simultaneously registered in the cloud system and recorded into Blockchain.

Figure 2 illustrates the steps operated in the design team model file and sheet file at different stages of development. 


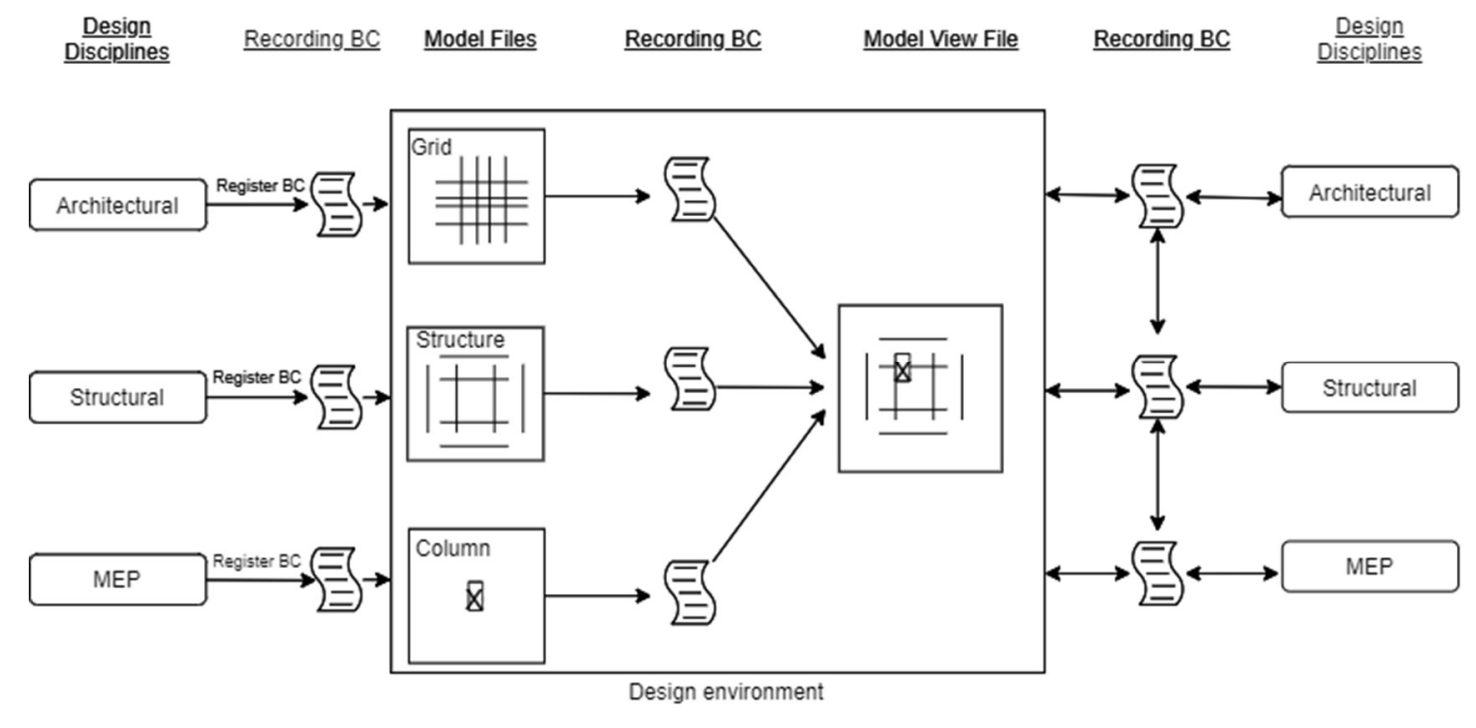

Fig. 2. Design team model file and Blockchain

The trial included three project disciplines, as mentioned below: (i) Discipline: A - 'Architect'; (ii) Discipline: S 'Structural Engineer' and (iii) Discipline: C - 'Client'.

\section{b. Smart contracts experiment}

We use Ethereum [64] to capture each model change to allow disciplines to build smart contracts with the attempt to develop a platform that can be used for project collaboration [65]. We use a Remix compiler, which is one of the tools used by Ethereum to simulate smart contract deployment and allows real-time debugging alongside all contract elements.

In our trial, we use data blocks for transactions using hash data signed with the discipline digital signature. We use a hash as a function that decodes data of the current block including the signature and creation details of the process that connects the blocks in the Blockchain. When a new block is created, the previous block's hash is combined with the current block's data, and a new hash is generated and signed with the user's digital signature.

Below we present a simple workflow that explains how the smart contract can be used to synchronize design records for multiple disciplines involved in the construction project:

- The Architect uploads an IFC file to the design record repository, which is a document library, and creates a URL (hyperlink).

- The Architect digitally signs the contract using a Blockchain API and creates a unique cryptographic hash for the document.

- The Architect assigns a Client to the system as a reviewer, and a smart contract proposal is generated in the allowed distributed ledger.

- The Client receives an email notification with a link to review the contract registration. The Client is asked to accept or reject the contract proposal.

- When the Client accepts the contract offer, they are prompted to sign the contract registration.

- After signing the contract record, the original smart contract is archived and a new fully fulfilled contract is created in the distributed ledger where the two parties enter the contract.
- The smart contract is synced with the contract record and combined with a reference to the hash attached to a document URL.

Step 1-Instalments: To run the Ethereum network, users need to register since the Ethereum network is a peer-to-peer network where one or more users validate blocks in order to operate the first network. In our case, the Blockchain network has a set of rules for the first block, also known as the genesis block. The genesis.json file provides a description for the Ethereum network. To begin the network, we built a client ID in the P2P network, followed by the wallet that will be used to process the transaction. The network is initiated and triggered in the following phase. The next step is to build a Smart Contract and to carry out this operation as a contract should be first written and compiled.

Step 2 - Discipline A (Architect): "Discipline A" has been registered into the system with its unique Blockchain ID and created a first bridge BIM model. All changes on the model are recorded to the Blockchain as seen in Table 1, which shows the first grid drawing after system records.

\begin{tabular}{|l|l|}
\hline Contract & Architect \\
\hline Input & First x-axis grid created \\
\hline Hash & $\underline{\text { a1075db5s54ysd9w86... }}$ \\
\hline Timestamp & $01.11 .2020-9: 30$ \\
\hline Previous Block & $\underline{0000000000074 a s d 56 \ldots}$ \\
\hline New Block & $\underline{0000000000074 d f f 43 \ldots \ldots}$ \\
\hline
\end{tabular}

Table 1: Seventh block of blockchain

Step 3 - Discipline S (Structural Engineer): The IFC file (A556-CAP-7000-S06-3D-S-1001.ifc) is shared with the structural design team, and the Blockchain is configured to keep consistent records as shown in Table 2. "Discipline S" starts to analyze the architectural drawing and to develop the structural design. During all of these stages, every step of design and file shares between design teams has been recorded into the Blockchain network. 


\begin{tabular}{|l|l|}
\hline Contract & Architect to Structural Engineer \\
\hline Input & $\begin{array}{l}\text { A556-CAP-7000-S06-3D-S-1001.ifc file } \\
\text { shared with the Structural Engineer }\end{array}$ \\
\hline Hash & $\underline{\mathbf{s} 4033 \mathrm{~d} 35 \mathrm{~s} 54 \mathrm{ysd} 9 \mathrm{w} 86 \ldots}$ \\
\hline Timestamp & $01.02 .2021-13: 48$ \\
\hline Previous Block & $\underline{0000000000085 \mathrm{~s} 3 \mathrm{~d} 56 \ldots}$ \\
\hline New Block & $\underline{0000000000085 \mathrm{dff} 43 \mathrm{a} \ldots}$ \\
\hline
\end{tabular}

Table 2: IFC file sharing between design team

Step 4 - Discipline C (Client): The IFC file can be shared with "Discipline C" at the same time to monitor the task process and for monitoring payment transactions. The Blockchain allows monitoring and tracking of the design data, as well as document records and file sharing between design teams at any time.

In the trial illustrated in Figure 3a, a smart contract has been created for the architect, structural engineer, and other design team to upload and change design records. A function has been specified to edit the variables when necessary, which only the design team was allowed to use for editing when a certain condition is met. The function was created at the end to capture the previous events and returns the design's records as well as design team address. In Figure 3b, it is shown that the smart contract was successfully executed.

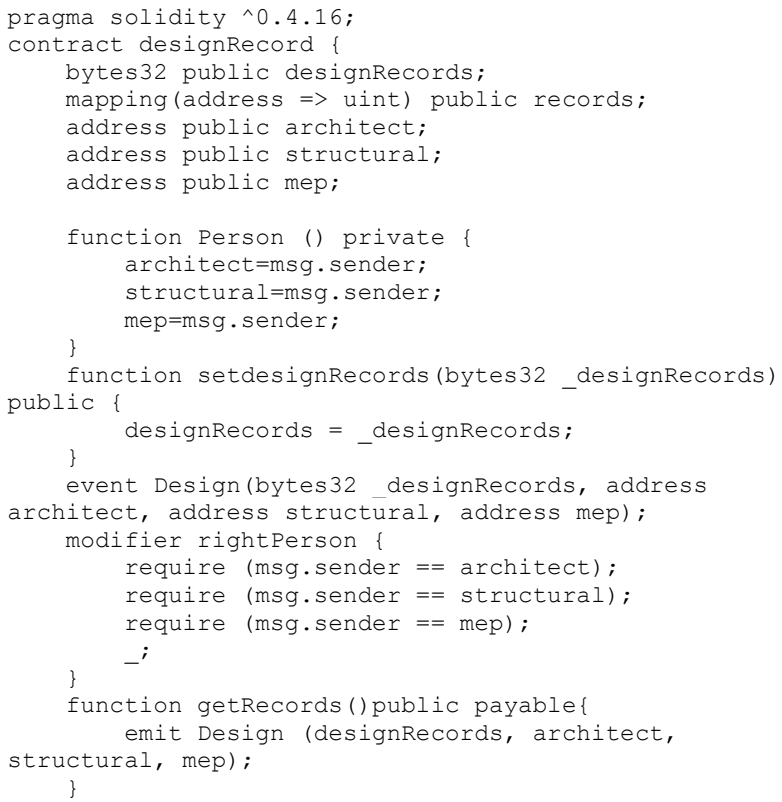

(a)

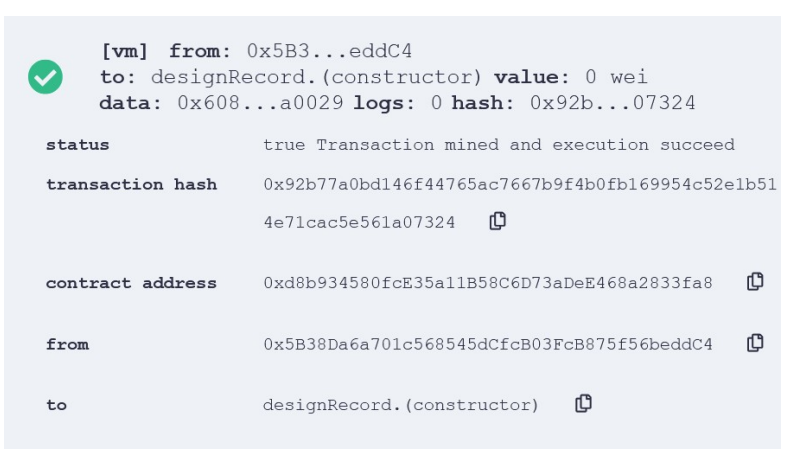

(b)

Fig. 3. a) Smart contract design records on Ethereum Remix. b) Execution of a Smart Contract

\section{ANALYSIS AND DISCUSSION}

The Blockchain and BIM integration is considered to be a solution for design collaboration facilitated with a secure interaction, data integrity, privacy, database management and data availability in a construction project. Blockchain enables the monitoring of users and their associated activities eliminating potential disputes between users in terms of duties and responsibilities. Each participant has its own set of project disciplines, roles, and responsibilities, resulting in a well-coordinated network of requirements.

The Blockchain platform's timestamp feature is ideal for recording any modifications to BIM models since it automates the design workflow and prevents design team members from making different alterations to the design. An additional benefit of an open and decentralised procedure is the decrease in the time necessary to develop the designs. With the use of blockchain, it will be possible to trace changes in BIM models from the contract phase to the postcontract phase. The blockchain maintains the proof of the history of all transactions and the chronological execution of each one, as well as storing ever-increasing amounts of information.

The decentralised structure of Blockchain simplifies users access to resources and knowledge located at different sources. Based on Blockchain ability to ensure traceability and immutable records, a secure environment is formed where participants can share BIM data more securely. A Blockchain database is distributed throughout the globe with individuals and resources. Blockchain systems support cooperation among consumers, contractors, and suppliers who come from various areas throughout the world. This can lead to the formation of communities of trust that leverage on the capability of, Blockchain to provide a stable, secure, and client-oriented collaborative platform where smart contracts can enable exchange of resources in a community of users.

Blockchain and BIM can also transform the needs and preferences of actors based on the services encoded in the digital twin process. The Blockchain implementation and participation management system for the entire BIM lifecycle from design to demolition connects the customer, contractor, real-time data and production, and employees can be exposed to users and disciplines via user interfaces in mobile terminals (tablets, VR-AR and smartphones).

With a Blockchain that stores lifecycle data as a distributed database in a global ledger including transaction records on the network, any change to the transaction list is monitored as each transmission has a timestamp and identifies a crypto hash. The controlled and hashed data is stored in the form of a ledger whose root is written in the block header with the help of cryptographic hashing algorithms. This ensures high transparency as parties sharing or examining files can be checked via the records made by the architect during the design process.

In terms of limitations, it should be reported that Blockchain is a technology that progressively expands with various industrial applications and further advances are expected which can impact our results. In addition, the conversion of contracts into codes for smart contracts can be also challenging as the history of smart contract activities cannot be changed while traditional contracts may, in practice, be modified with the consent of all parties. 


\section{CONCLUSION}

BIM represents a "pillar' for construction digitalization that can support the collaborative work in the construction sector. To enable transition to digital twinning, security problems and issues arising during multi-disciplinary data sharing, delays in the project or payment as a result of the disagreements in the duties, need also to be addressed by technology.

A solution addressing such limitations is to record all kinds of transactions and activities in a data environment using the Blockchain technology. Blockchain can issue a unique record that cannot be edited or changed by participative project disciplines. Such functionality is in particular beneficial when disputes appear, as Blockchain artefacts can represent real evidences on behalf of roles and responsibilities including file sharing and actions operated in the file.

Blockchain has also the ability transform the construction industry and pave the way towards a higher order of digitalisation across industries. At the supply chain level in the construction industry, Blockchain increases process traceability and keeps the originality of the material or service. Other benefits include usability, trust, safety, scalability and data storage. Code control compatibility for BIM workflows is also supported by Blockchain based on programming simplicity, versatility, and user-defined smart contracts leading to a viable and highly safe automation. A Blockchain framework for construction projects can bring more reliability and high traceability and the precision of cost and timing estimates.

Future research will focus on developing more complex examples with evidences from real case studies in the attempt to combine Blockchain, machine learning and Internet-ofThings for construction automation.

\section{REFERENCES}

[1] F. Elghaish, S. Abrishami, and M. R. Hosseini, "Integrated project delivery with blockchain: An automated financial system," Autom. Constr., vol. 114, no. March, p. 103182, 2020, doi: 10.1016/j.autcon.2020.103182.

[2] "slaughter1998.pdf."

[3] M. Schluse, M. Priggemeyer, L. Atorf, and J. Rossmann, "Experimentable Digital Twins-Streamlining Simulation-Based Systems Engineering for Industry 4.0," IEEE Trans. Ind. Informatics, vol. 14, no. 4, pp. 1722-1731, 2018, doi: 10.1109/TII.2018.2804917.

[4] H. R. Hasan et al., "A Blockchain-Based Approach for the Creation of Digital Twins," IEEE Access, vol. 8, pp. 3411334126, 2020, doi: 10.1109/ACCESS.2020.2974810

[5] S. Aheleroff, X. Xu, R. Y. Zhong, and Y. Lu, "Digital Twin as a Service (DTaaS) in Industry 4.0: An Architecture Reference Model," Adv. Eng. Informatics, vol. 47, no. December 2020, p. 101225, 2021, doi: 10.1016/j.aei.2020.101225.

[6] M. Ardolino, M. Rapaccini, N. Saccani, P. Gaiardelli, G. Crespi, and C. Ruggeri, "The role of digital technologies for the service transformation of industrial companies," Int. J. Prod. Res., vol. 56, no. 6, pp. 2116-2132, 2018, doi: 10.1080/00207543.2017.1324224.

[7] C. M. Eastman, P. Teicholz, R. Sacks, and K. Liston, A Guide to Building Information Modeling for Owners, Managers, Architects, Engineers, Contractors, and Fabricators. 2008.

[8] J. Gardan, "Additive manufacturing technologies: State of the art and trends," Int. J. Prod. Res., vol. 54, no. 10, pp. 3118-3132, 2016, doi: 10.1080/00207543.2015.1115909.

[9] F. Xue and W. Lu, “A semantic differential transaction approach to minimizing information redundancy for BIM and blockchain integration," Autom. Constr., vol. 118, no. February, p. 103270, 2020, doi: 10.1016/j.autcon.2020.103270.

[10] M. Krämer and Z. Besenyoi, "Towards Digitalization of Building Operations with BIM," IOP Conf. Ser. Mater. Sci. Eng., vol. 365 , no. 2, 2018, doi: 10.1088/1757-899X/365/2/022067.

[11] D. Lee, S. H. Lee, N. Masoud, M. S. Krishnan, and V. C. Li, "Integrated digital twin and blockchain framework to support accountable information sharing in construction projects," Autom. Constr., vol. 127, no. March, p. 103688, 2021, doi: 10.1016/j.autcon.2021.103688.

[12] Q. Lu et al., "Developing a Digital Twin at Building and City Levels: Case Study of West Cambridge Campus," J. Manag. Eng., vol. 36, no. 3, p. 05020004, 2020, doi: 10.1061/(asce)me.1943-5479.0000763.

[13] M. Mathews, B. Bowe, and D. Robles, "BIM+Blockchain: A Solution to the Trust Problem in Collaboration? | Enhanced Reader," CITA BIM Gather. 2017, 2017, [Online]. Available: moz-extension://db27a32d-d4bf-c540-b67f-

$85 \mathrm{ad} 2 \mathrm{caf} 9 \mathrm{dba} /$ enhanced-

reader.html?openApp\&pdf $=$ https $\% 3 \mathrm{~A} \% 2 \mathrm{~F} \% 2$ Farrow.tudublin.ie $\% 2$ Fcgi $\% 2$ Fviewcontent.cgi $\% 3$ Farticle $\% 3$ D $1032 \% 26$ context $\% 3$ Dbescharcon

[14] R. Alonso, M. Borras, R. H. E. M. Koppelaar, A. Lodigiani, E. Loscos, and E. Yöntem, "SPHERE : BIM Digital Twin Platform $\dagger$,” pp. 1-6, 2019, doi: 10.3390/proceedings2019020009

[15] M. Bevilacqua et al., "Digital twin reference model development to prevent operators' risk in process plants," Sustain., vol. 12, no. 3, pp. 1-17, 2020, doi: 10.3390/su12031088.

[16] S. Azhar, A. Nadeem, J. Y. N. Mok, and B. H. Y. Leung, "Building Information Modeling (BIM): A New Paradigm for Visual Interactive Modeling and Simulation for Construction Projects," First Int. Conf. Constr. Dev. Ctries., no. November 2017, pp. 435-446, 2008.

[17] S. H. Khajavi, N. H. Motlagh, A. Jaribion, L. C. Werner, and J. Holmstrom, "Digital Twin: Vision, benefits, boundaries, and creation for buildings," IEEE Access, vol. 7, pp. 147406-147419, 2019, doi: 10.1109/ACCESS.2019.2946515.

[18] C. Boje, A. Guerriero, S. Kubicki, and Y. Rezgui, "Towards a semantic Construction Digital Twin: Directions for future research," Autom. Constr., vol. 114, no. November 2019, p 103179, 2020, doi: 10.1016/j.autcon.2020.103179.

[19] J. M. Sardroud, M. Mehdizadehtavasani, A. Khorramabadi, and A. Ranjbardar, "Barriers analysis to effective implementation of BIM in the construction industry," ISARC 2018 - 35th Int. Symp. Autom. Robot. Constr. Int. AEC/FM Hackathon Futur. Build. Things, no. July, 2018, doi: 10.22260/isarc2018/0009.

[20] E. A. Parn and D. Edwards, "Cyber threats confronting the digital built environment: Common data environment vulnerabilities and block chain deterrence," Eng. Constr. Archit. Manag., vol. 26, no. 2, pp. 245-266, 2019, doi: 10.1108/ECAM-03-2018-0101.

[21] N. O. Nawari and S. Ravindran, "Blockchain and the built environment: Potentials and limitations," J. Build. Eng., vol. 25, no. June, 2019, doi: 10.1016/j.jobe.2019.100832.

[22] J. Garzik and J. C. Donnelly, Blockchain 101: An Introduction to the Future, 1st ed., vol. 2. Elsevier Inc., 2018.

[23] Smart Contracts Alliance, "Smart Contracts : 12 Use Cases for Business \& Beyond," Chamb. Digit. Commer., 2016.

[24] "Smart Contracts."

http://www.fon.hum.uva.nl/rob/Courses/InformationInSpeech/C DROM/Literature/LOTwinterschool2006/szabo.best.vwh.net/sma rt.contracts.html (accessed May 15, 2020).

[25] A. Bahga and V. K. Madisetti, "Blockchain Platform for Industrial Internet of Things," pp. 533-546, 2016, doi: 10.4236/jsea.2016.910036.

[26] H. Vranken, "Sustainability of bitcoin and blockchains," Curr Opin. Environ. Sustain., vol. 28, pp. 1-9, 2017, doi: 10.1016/j.cosust.2017.04.011

[27] W. Zou et al., "Smart Contract Development: Challenges and Opportunities," IEEE Trans. Softw. Eng., vol. 5589, no. March 2018, pp. 1-1, 2019, doi: 10.1109/tse.2019.2942301.

[28] M. R. Prasad, R. L. Naik, and V. Bapuji, "Cloud Computing : Research Issues and Implications," Int. J. Cloud Comput. Serv. Sci., 2013, doi: 10.11591/closer.v2i2.1963. 
[29] W. Venters and E. A. Whitley, "A critical review of cloud computing: Researching desires and realities," Journal of Information Technology. 2012, doi: 10.1057/jit.2012.17.

[30] M. M. Islam, S. Morshed, and P. Goswami, "Cloud Computing: A Survey on its limitations and Potential Solutions," Int. J. Comput. Sci. Issues, vol. 10, no. 4, pp. 159-163, 2013.

[31] B. Hay, K. Nance, and M. Bishop, "Storm clouds rising: Security challenges for IaaS cloud computing," Proc. Annu. Hawaii Int. Conf. Syst. Sci., pp. 1-7, 2011, doi: 10.1109/HICSS.2011.386.

[32] S. A. Bello et al., "Cloud computing in construction industry: Use cases, benefits and challenges," Autom. Constr., vol. 122, p. 103441, 2021, doi: 10.1016/j.autcon.2020.103441.

[33] C. V. N. U. B. Murthy, M. L. Shri, S. Kadry, and S. Lim, "Blockchain Based Cloud Computing: Architecture and Research Challenges," IEEE Access, vol. 8, no. November, pp. 205190 205205, 2020, doi: 10.1109/access.2020.3036812.

[34] R. Patriarca, J. Bergström, G. Di Gravio, and F. Costantino, "Resilience engineering: Current status of the research and future challenges," Saf. Sci., vol. 102, no. December 2016, pp. 79-100, 2018, doi: 10.1016/j.ssci.2017.10.005.

[35] B. Keskin, B. Salman, and B. Ozorhon, "Airport project delivery within BIM-centric construction technology ecosystems," Eng. Constr. Archit. Manag., 2020, doi: 10.1108/ECAM-11-20190625.

[36] C. M. Eastman, P. Teicholz, R. Sacks, and K. Liston, "Interoperability (Chapter 3)," in BIM handbook: a guide to building information modeling for owners, managers, designers, engineers, and contractors, 2011.

[37] K. Christidis and M. Devetsikiotis, "Blockchains and Smart Contracts for the Internet of Things," IEEE Access, vol. 4, pp. 2292-2303, 2016, doi: 10.1109/ACCESS.2016.2566339.

[38] N. Kshetri and J. Voas, "Blockchain in Developing Countries," IT Prof., vol. 20, no. 2, pp. 11-14, 2018, doi:

10.1109/MITP.2018.021921645.

[39] J. Mason, "Intelligent Contracts and the Construction Industry," J. Leg. Aff. Disput. Resolut. Eng. Constr., vol. 9, no. 3, p. 04517012, Aug. 2017, doi: 10.1061/(asce)la.1943-4170.0000233.

[40] M. Mathews, D. Robles, and B. Bowe, "BIM+Blockchain: A Solution to the Trust Problem in Collaboration?," CITA BIM Gather. 2017, p. 11, 2017, [Online]. Available: https://arrow.tudublin.ie/bescharcon.

[41] S. Smith and L. O'rourke, "Exploring the potential of Blockchain technology for the UK Construction industry - 2019," no. April, 2019.

[42] T. Y. Lam and B. Dongol, "A blockchain-enabled e-learning platform," Interact. Learn. Environ., 2020, doi: $10.1080 / 10494820.2020 .1716022$.

[43] A. A. Hijazi, S. Perera, A. Alashwal, and R. N. Calheiros, "Blockchain Adoption in Construction Supply Chain : A Review of Studies Blockchain Adoption in Construction Supply Chain: A Review of Studies Across Multiple Sectors," CIB World Build. Congr. 2019, no. June, 2019, [Online]. Available: https://www.researchgate.net/publication/333879452_Blockchain _Adoption_in_Construction_Supply_Chain_A_Review_of_Studi es_Across_Multiple_Sectors.

[44] N. Mohamed and J. Al-Jaroodi, "Applying blockchain in industry 4.0 applications," 2019 IEEE 9th Annu. Comput. Commun. Work. Conf. CCWC 2019, pp. 852-858, 2019, doi:

10.1109/CCWC.2019.8666558

[45] H. M. Kim and M. Laskowski, "Toward an ontology-driven blockchain design for supply-chain provenance," Intell. Syst. Accounting, Financ. Manag., vol. 25, no. 1, pp. 18-27, 2018, doi: 10.1002/isaf.1424

[46] C. Mandolla, A. M. Petruzzelli, G. Percoco, and A. Urbinati, "Building a digital twin for additive manufacturing through the exploitation of blockchain: A case analysis of the aircraft industry," Comput. Ind., vol. 109, pp. 134-152, 2019, doi: 10.1016/j.compind.2019.04.011.

[47] N. Deepa et al., "A survey on blockchain for big data:
Approaches, opportunities, and future directions," arXiv, pp. 120, 2020.

[48] D. B. Rawat, R. Doku, and M. Garuba, "Cybersecurity in Big Data Era: From Securing Big Data to Data-Driven Security," IEEE Trans. Serv. Comput., vol. 1374, no. c, pp. 1-1, 2019, doi: 10.1109/tsc.2019.2907247.

[49] A. A. Siyal, A. Z. Junejo, M. Zawish, K. Ahmed, A. Khalil, and G. Soursou, "Applications of blockchain technology in medicine and healthcare: Challenges and future perspectives," Cryptography, vol. 3, no. 1, pp. 1-16, 2019, doi: 10.3390/cryptography3010003.

[50] A. Madni, C. Madni, and S. Lucero, "Leveraging Digital Twin Technology in Model-Based Systems Engineering," Systems, vol. 7, no. 1, p. 7, 2019, doi: 10.3390/systems7010007.

[51] M. Macchi, I. Roda, E. Negri, and L. Fumagalli, "Exploring the role of Digital Twin for Asset Lifecycle Management," IFACPapersOnLine, vol. 51, no. 11, pp. 790-795, 2018, doi: 10.1016/j.ifacol.2018.08.415.

[52] F. Tao et al., "Digital twin and blockchain enhanced smart manufacturing service collaboration and management," J. Manuf. Syst., no. February, 2020, doi: 10.1016/j.jmsy.2020.11.008.

[53] I. Yaqoob, K. Salah, M. Uddin, R. Jayaraman, M. Omar, and M. Imran, "Blockchain for Digital Twins: Recent Advances and Future Research Challenges," IEEE Netw., vol. 34, no. 5, pp. 290-298, 2020, doi: 10.1109/MNET.001.1900661.

[54] K. Y. H. Lim, P. Zheng, and C. H. Chen, "A state-of-the-art survey of Digital Twin: techniques, engineering product lifecycle management and business innovation perspectives," J. Intell. Manuf., vol. 31, no. 6, pp. 1313-1337, 2020, doi: 10.1007/s10845-019-01512-w.

[55] D. Guo et al., "A framework for personalized production based on digital twin, blockchain and additive manufacturing in the context of Industry 4.0," IEEE Int. Conf. Autom. Sci. Eng., vol. 2020-Augus, pp. 1181-1186, 2020, doi: 10.1109/CASE48305.2020.9216732.

[56] J. Angelis and E. Ribeiro da Silva, "Blockchain adoption: A value driver perspective," Bus. Horiz., vol. 62, no. 3, pp. 307314, 2019, doi: 10.1016/j.bushor.2018.12.001.

[57] M. Kuhn, F. Funk, G. Zhang, and J. Franke, "Blockchain-based application for the traceability of complex assembly structures," J. Manuf. Syst., vol. 59, no. December 2020, pp. 617-630, 2021, doi: 10.1016/j.jmsy.2021.04.013.

[58] K. Wang et al., "Simulation-Based Digital Twin Development for Blockchain Enabled End-To-End Industrial Hemp Supply Chain Risk Management," arXiv, pp. 1-11, 2020.

[59] H. Huang, W. Kong, S. Zhou, Z. Zheng, and S. Guo, "A Survey of State-of-the-Art on Blockchains: Theories, Modelings, and Tools," arXiv, 2020.

[60] Y. Chen and C. Bellavitis, "Blockchain disruption and decentralized finance: The rise of decentralized business models," J. Bus. Ventur. Insights, vol. 13, no. October 2019, p. e00151, 2020, doi: 10.1016/j.jbvi.2019.e00151.

[61] J. Wang, P. Wu, X. Wang, and W. Shou, "The outlook of blockchain technology for construction engineering management," vol. 4, no. 1, pp. 67-75, 2017, doi: 10.15302/JFEM-2017006.

[62] U. Ahmed, I. Petri, O. Rana, I. Raza, and S. A. Hussain, "Federating Cloud Systems for Collaborative Construction and Engineering," IEEE Access, vol. 8, pp. 79908-79919, 2020, doi: 10.1109/ACCESS.2020.2990233.

[63] T. H. Beach, O. F. Rana, Y. Y. Rezgui, and M. Parashar, "Cloud computing for the architecture, engineering \& construction sector: Requirements, prototype \& experience," J. Cloud Comput., vol. 2, no. 1, 2013, doi: 10.1186/2192-113X-2-8.

[64] G. Wood, "Ethereum: a secure decentralised generalised transaction ledger," Ethereum Proj. Yellow Pap., 2014.

[65] C. Dannen, Introducing ethereum and solidity: Foundations of cryptocurrency and blockchain programming for beginners. 2017. 